Gut and Liver, Vol. 9, No. 3, May 2015, pp. 259-260

\title{
There Is No Reason to Delay Helicobacter pylori Eradication after Treatment for Upper Gastrointestinal Bleeding
}

\author{
Sun-Young Lee \\ Department of Internal Medicine, Konkuk University School of Medicine, Seoul, Korea
}

See "Helicobacter pylori Eradication within 120 Days Is Associated with Decreased Complicated Recurrent Peptic Ulcers in Peptic Ulcer Bleeding Patients" by Shen Shong Chang, et al. on page 346, Vol. 9. No. 3, 2015

Helicobacter pylori is a major cause of peptic ulcer disease (PUD) recurrence, and thus eradication is essential for the treatment of PUD patients with $H$. pylori infection. ${ }^{1}$ Although revised guidelines on $H$. pylori infection differ between countries due to differences in national health insurance systems, $H$. pylori eradication is recommended for PUD in the Korean, Japanese, and Chinese guidelines. ${ }^{2}$ Data from the Cochrane database show that H. pylori eradication is more effective than antisecretory therapy for preventing recurrent PUD-associated bleeding. ${ }^{3}$ Consequently, all patients should be tested for $H$. pylori infection after hemostasis for PUD-associated bleeding, and eradication should be performed in $H$. pylori-positive patients. However, there is no evidence regarding the optimal timing of $H$. pylori eradication in these subjects.

In a Taiwanese study by Drs. Chang and $\mathrm{Hu}^{4}$ reported in this issue of Gut and Liver, H. pylori eradication within 120 days (i.e., early $H$. pylori eradication therapy) was significantly associated with reduced complicated recurrent PUD in patients with bleeding control. Using the Taiwan National Health Insurance Research Database, those authors found that patients in the late $H$. pylori eradication therapy group (i.e., H. pylori eradication after 120 days) who were admitted and treated for PUD-associated bleeding in Taiwan between 2000 and 2010 had a higher rate of complicated recurrent PUD. That study is the first large-sized comparison between early and late timing of $H$. pylori eradication therapy in PUD after bleeding control.

Chang and $\mathrm{Hu}^{4}$ analyzed possible confounding factors from various viewpoints. They excluded patients under the age of 20 years, those with prior gastrectomies or vagotomies, those with cerebral vascular disease, liver cirrhosis, or chronic kidney disease, and those diagnosed with gastric cancer or Zollinger-
Ellison syndrome. With regard to medications, patients were defined as users of proton pump inhibitors (PPIs), histamine 2 $\left(\mathrm{H}_{2}\right)$-blockers, aspirin, nonsteroidal anti-inflammatory drugs (NSAIDs), cyclooxygenase 2-specific inhibitors, steroids, clopidogrel, ticlopidine, and warfarin. In a recent study of 522 PUD patients with upper gastrointestinal bleeding, rebleeding was more frequent in patients on aspirin medication and after endoscopic hemostasis lasting longer than 13.5 minutes. ${ }^{5}$ Notably, in the study by Chang and $\mathrm{Hu},{ }^{4}$ the proportion of patients who used PPIs, $\mathrm{H}_{2}$ blockers, or NSAIDs was significantly lower in the early eradication group than in the late eradication group. The authors explained that participants taking PPIs or $\mathrm{H}_{2}$ blockers may have higher risk of PUD-associated bleeding, but this issue requires further clarification. Interestingly, of the significant factors, late $H$. pylori eradication therapy was an independent risk factor for rehospitalization on multivariate analysis. On the other hand, time delays of more than 1 year did not increase the risk of complicated recurrent PUD.

With regard to the diagnosis for $H$. pylori infection, patients with upper gastrointestinal bleeding frequently exhibit falsenegative findings on $H$. pylori infection. Furthermore, testing for $H$. pylori is affected by concomitant medications such as NSAIDs, aspirin, or PPIs. These finding might be attributable to the low percentage of patients who underwent eradication in that study, only 2,463 patients of the 12,686 patients hospitalized with PUD-associated bleeding underwent $H$. pylori eradication. ${ }^{4}$ Neither second look endoscopy nor ${ }^{13} \mathrm{C}$-urea breath test was performed to confirm $H$. pylori status in patients who were initially negative for $H$. pylori by diagnostic testing. Moreover, the diagnosis of $H$. pylori infection in the study of Chang and $\mathrm{Hu}^{4}$ was based on the results of a rapid urease test or histologi-

Correspondence to: Sun-Young Lee

Department of Internal Medicine, Konkuk University School of Medicine, 120 Neungdong-ro, Gwangjin-gu, Seoul 143-729, Korea

Tel: +82-2-2030-7505, Fax: +82-2-2030-7748, E-mail: sunyoung@kuh.ac.kr

pISSN 1976-2283 eISSN 2005-1212 http://dx.doi.org/10.5009/gnl14499

@ This is an Open Access article distributed under the terms of the Creative Commons Attribution Non-Commercial License (http://creativecommons.org/licenses/by-nc/3.0) which permits unrestricted non-commercial use, distribution, and reproduction in any medium, provided the original work is properly cited. 
cal assessment using hematoxylin and eosin staining following $H$. pylori eradication therapy. There was no uniformity in the $H$. pylori diagnostic tests before and after the eradication, and various factors that affect the eradication rate (i.e., adherence of patient, adverse effect, genetic polymorphism related to drug metabolism, antibiotics resistance, reinfection, recrudescence, duration of medication, dose of antibiotics, interval of intake relation to meals, regimen, formulation, manufacturers, other medications, time of follow-up test, and diagnostic method) were not considered. A serum $H$. pylori antibody test was not performed in that study, despite a decrease relative to the initial antibody titer of more than 50\% after 6 to 12 months being considered the most accurate method for determining successful eradication.

Finally, care should be taken when interpreting the findings of Chang and $\mathrm{Hu}^{4}$ since they used "rehospitalization" as a primary outcome, rather than "rebleeding" or "mortality." As noted by the authors when discussing the limitations of their study, differences in physician behavior and admission criteria for PUDs were also potential confounders. However, only the risk of rehospitalization for endoscopically confirmed complicated recurrent PUD was analyzed.

In summary, there is no reason to delay $H$. pylori eradication in patients with PUD-associated bleeding after hemostasis. If the initial diagnosis for $H$. pylori infection is negative at the time of PUD-associated bleeding, follow-up tests using a noninvasive method are recommended to exclude false-negative findings for $H$. pylori infection. Given the significance of $H$. pylori infection in PUD-associated bleeding, ${ }^{6}$ eradication should be performed within 120 days after the achievement of bleeding control.

\section{CONFLICTS OF INTEREST}

No potential conflict of interest relevant to this article was reported.

\section{ACKNOWLEDGEMENTS}

This work was supported by Konkuk University.

\section{REFERENCES}

1. Kim JJ, Kim N, Lee BH, et al. Risk factors for development and recurrence of peptic ulcer disease. Korean J Gastroenterol 2010;56:220-228.

2. Lee SY. Current progress toward eradicating Helicobacter pylori in East Asian countries: differences in the 2013 revised guidelines between China, Japan, and South Korea. World J Gastroenterol 2014;20:1493-1502.

3. Gisbert JP, Khorrami S, Carballo F, Calvet X, Gené E, DominguezMuñoz JE. H. pylori eradication therapy vs. antisecretory noneradication therapy (with or without long-term maintenance antisecretory therapy) for the prevention of recurrent bleeding from peptic ulcer. Cochrane Database Syst Rev 2004;(2):CD004062.

4. Chang SS, Hu HY. Helicobacter pylori eradication within 120 days is associated with decreased complicated recurrent peptic ulcers in peptic ulcer bleeding patients. Gut Liver 2015;9:346-352.

5. Hong MJ, Lee SY, Kim JH, et al. Rebleeding after initial endoscopic hemostasis in peptic ulcer disease. J Korean Med Sci 2014;29:1411-1415.

6. Konturek SJ, Konturek PC, Konturek JW, et al. Helicobacter pylori and its involvement in gastritis and peptic ulcer formation. J Physiol Pharmacol 2006;57 Suppl 3:29-50. 